\title{
Modelling and Control of a 2-DOF Planar Parallel Manipulator for Semiconductor Packaging Systems
}

\author{
Jacob W.F. Cheung ${ }^{\dagger}$ and Y.S. Hung ${ }^{\ddagger}$ \\ ${ }^{\dagger}$ Motion Control and Systems Laboratory, Research \& Development Department, \\ ASM Assembly Automation Ltd., 3/F Watson Centre, 16 Kung Yip Street, Kwai Chung, \\ New Territories, Hong Kong. \\ Department of Electrical and Electronic Engineering, The University of Hong Kong, \\ Pokfulam Road, Hong Kong.
}

\begin{abstract}
A novel direct-drive planar parallel manipulator for high-speed and high-precision semiconductor packaging systems is presented. High precision kinematics design, significant reduction on moving mass and driving power of the actuators over traditional XY motion stages are the benefits of the proposed manipulator. The mathematical model of the manipulator is obtained using the Newton-Euler method and a practical model-based control design approach is employed to design the PID computed-torque controller. Experimental results demonstrate that the proposed planar parallel manipulator has significant improvements on motion performance in terms of positioning accuracy, settling time and stability when compared with traditional XY stages. This shows that the proposed planar parallel manipulator can provide a superior alternative for replacing traditional XY motion stages in high precision low-payload applications.
\end{abstract}

\section{INTRODUCTION}

Parallel manipulators have been used in various kinds of industries, after being proposed by Gough [1] and Stewart [2]. The Stewart platform has been modified and improved to the modern Hexapod machine, which is mainly used for multi-axis CNC machining centre. A class of parallel manipulator known as the Delta robot has been proposed by Clavel [3]. Since Delta robots have multiple DOF (degreeof-freedom) passive joints (universal joints and ball-andsocket joints), the positional accuracy is hard to assure.

In this paper, we will study a 2-DOF parallel mechanism as an alternative to the traditional XY-stage for highprecision, low-payload motion aimed at semiconductor packaging applications. End-point accuracy and repeatability are essential for high-precision positioning mechanism used in semiconductor packaging systems to assure product quality. We will show that compared to the traditional $\mathrm{XY}$ motion stages, the advantages of the proposed planar parallel manipulator include significant improvements in the position accuracy and settling time, light moving mass of the mechanism and reduction in the motor driving power.

Feedback control plays a crucial role in achieving the

This project is funded and supported by ASM Assembly Automation Ltd., Hong Kong. For further information, please contact jacob.cheung@asmpt.com or yshung@eee.hku.hk. The second author is supported by a CRCG grant from The University of Hong Kong. required position accuracy. A joint-based controller with acceleration feedback was proposed by Chiacchio et al. [4] using a state-variable filter with linear feedforward compensation. A computed torque plus $H_{\infty}$ compensation method has been developed by Lee and Cheng [5] to deal with the manipulator payload and actuator uncertainties. Another approach was proposed by Lin and Brandt [6] using optimal control method. However, these methods are not suitable in our case as the payload of semiconductor devices is negligible compared to the mass of the manipulator and the characteristic of the linear actuators can be readily determined. A non-parametric model robust control design method was proposed by Gonzalez and Aguilar [7] using external signals to excite the manipulator for measuring the output position. A simple PD with gravity controller was proposed by Ghorbel et al. [8] to perform the set point control of closed-chain mechanism. This approach has been modified by Pierrot et al. [9] and [10] to control a redundant mechanism. A predictive functional control approach was proposed in [11] to control the manipulator presented in [10]. We will use a model-based design approach to design the required high-precision position control system.

The paper is organized as follows. In Section II, the kinematics and dynamic models of the proposed planar parallel manipulator are given. Section III describes the model-based approach of the PID computed-torque control system design. A prototype of the parallel manipulator has been constructed and the experimental setup of the prototype together with a high-precision laser displacement measurement system is described in Section IV. The results obtained from the experiments performed on the prototype are given in Section V. Section VI contains some concluding remarks.

\section{KINEMATICS AND DYNAMIC MODELS}

\section{A. Kinematics model}

Fig. 1 shows the kinematics design of the proposed 2DOF planar parallel manipulator. The manipulator provides 2-DOF of translation on the XY plane, and is driven by a linear miniature servomotor pair located at $\left(d_{l}, d_{3}\right)$ along the $\mathrm{X}$-axis. The linear actuators are coupled to a 2-DOF 
triangular planar mechanism which can be used to place the point $G(x, y)$ at desired positions on the XY-plane. Note that the small platform at the outer joint of the triangular mechanism in Fig. 1 is constrained by a belt mechanism to have only translational motion but not rotation on the XYplane.

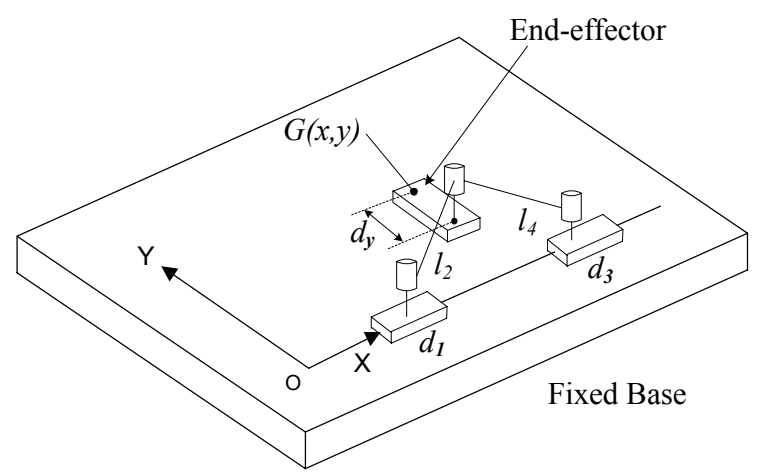

Fig. 1. Kinematics design of the 2-DOF planar parallel manipulator.

Let the positions $d_{1}$ and $d_{3}$ of the linear actuators satisfy:

$$
0<d_{1 \min } \leq d_{1} \leq d_{1 \max }<d_{3 \min } \leq d_{3} \leq d_{3 \max } .
$$

In the case when $l_{2}=l_{4}=l$, the forward kinematics can be determined by relating the position of $G(x, y)$ to the actuator positions as

$$
\begin{aligned}
& x=\left(d_{1}+d_{3}\right) / 2 \\
& y=\sqrt{l^{2}-\left(d_{3}-d_{1}\right)^{2} / 4}+d_{y}
\end{aligned}
$$

To obtain the inverse kinematics, we note that $d_{1}<d_{3}$. The positions of the linear actuators can be solved from (1) as

$$
\begin{aligned}
& d_{1}=x-\sqrt{l^{2}-\left(y-d_{y}\right)^{2}} \\
& d_{3}=x+\sqrt{l^{2}-\left(y-d_{y}\right)^{2}}
\end{aligned}
$$

\section{B. Dynamic model}

Fig. 2 shows the free-body diagram of the 2-DOF parallel manipulator. In Fig. 2, $m_{1}$ and $m_{3}$ are the masses of the linear actuators and $m_{2}$ and $m_{4}$ are the masses of the linkages. The inertia of link 2 and link 4 about the centre of mass are $I_{2}$ and $I_{4}$ respectively. The dynamic model of the planar parallel manipulator can be expressed in the form [14]

$$
F_{p}=M_{p}(q) \ddot{q}+C_{p}(q, \dot{q})
$$

where $F_{p}$ denotes the actuating force vector, $q$ represents the generalized coordinates vector, $M_{p}$ represents the mass matrix of the planar manipulator and $C_{p}$ represents the velocity coupling vector.

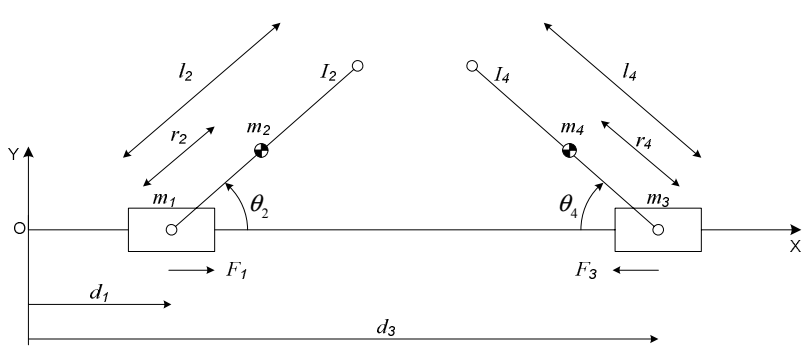

Fig. 2. Free-body diagram of the planar parallel manipulator.

In the parallel manipulator, the inputs are the force exerted by the linear actuators,

$$
F_{p}=\left[\begin{array}{ll}
F_{1} & F_{3}
\end{array}\right]^{T}
$$

and the generalized joint variable vector is

$$
q=\left[\begin{array}{ll}
d_{1} & d_{3}
\end{array}\right]^{T}
$$

Because of the closed kinematics structure and also by the symmetry of the linkages, the joint variables of the passive revolute joints can be expressed in terms of $q$ as:

$$
\theta_{2}=\theta_{4}=\cos ^{-1}\left(\left(d_{3}-d_{1}\right) / 2 l\right)
$$

The parameter matrices in (3) are given by:

$$
\begin{aligned}
& M_{p}=\left[\begin{array}{ll}
M_{11} & M_{12} \\
M_{21} & M_{22}
\end{array}\right] \\
& C_{p}=\left[\begin{array}{ll}
C_{1} & C_{2}
\end{array}\right]^{T}
\end{aligned}
$$

$$
\begin{aligned}
M_{11}= & m_{1}+m_{2}-\frac{m_{2} r_{2}}{l_{2}}+\frac{m_{4} r_{4}^{2}+I_{4}+m_{2} r_{2}^{2}+I_{2}}{4 l_{2}{ }^{2} s^{2} \theta_{2}} \\
& -\frac{m_{p} h_{2} c \theta_{2}}{2 s \theta_{2}}-\frac{m_{p}}{4} \\
M_{12}= & \frac{m_{4} r_{4}+m_{2} r_{2}}{2 l_{2}}-\frac{m_{4} r_{4}{ }^{2}+I_{4}+m_{2} r_{2}{ }^{2}+I_{2}}{4 l_{2}{ }^{2} s^{2} \theta_{2}} \\
& +\frac{m_{p} h_{2} c \theta_{2}}{2 s \theta_{2}}-\frac{m_{p}}{4} \\
M_{21} & =-M_{12}
\end{aligned}
$$

$$
\begin{aligned}
M_{22}= & -m_{3}-m_{4}+\frac{m_{4} r_{4}}{l_{2}} \\
& -\frac{m_{4} r_{4}{ }^{2}+I_{4}+m_{2} r_{2}{ }^{2}+I_{2}}{4 l_{2}{ }^{2} s^{2} \theta_{2}}+\frac{m_{p} h_{2} c \theta_{2}}{2 s \theta_{2}}+\frac{m_{p}}{4} \\
C_{1}=C_{2}= & -\frac{\left(m_{4} r_{4}{ }^{2}+I_{4}+m_{2} r_{2}{ }^{2}+I_{2}\right) c \theta_{2}\left(\dot{d}_{1}-\dot{d}_{3}\right)^{2}}{8 l_{2}{ }^{3} s^{4} \theta_{2}} \\
& +\frac{m_{p} h_{1} c \theta_{2}}{2 s \theta_{2}}
\end{aligned}
$$




$$
\begin{gathered}
h_{1}=-\frac{\left(d_{3}-d_{1}\right)^{2}\left(\dot{d}_{3}-\dot{d}_{1}\right)^{2}}{16\left(l^{2}-\left(d_{3}-d_{1}\right)^{2} / 4\right)^{3 / 2}}-\frac{\left(\dot{d}_{3}-\dot{d}_{1}\right)^{2}}{4 \sqrt{l^{2}-\left(d_{3}-d_{1}\right)^{2} / 4}} \\
h_{2}=-\frac{\left(d_{3}-d_{1}\right)}{4 \sqrt{l_{2}^{2}-\left(d_{3}-d_{1}\right)^{2} / 4}}
\end{gathered}
$$

where $m_{p}$ is the mass of the end-effector . Equations (3) to (14) provide a complete dynamic model of the proposed planar manipulator.

\section{COMPUTED-TORque CONTROL DESIGN}

The dynamic model (3) of the proposed planar manipulator developed in Section II is used for designing the controller. First, feedback linearization is applied to convert the nonlinear manipulator model into a linear model. Suppose the actuating force vector $F_{p}$ is generated by

$$
F_{p}=M_{p}(q) u+C_{p}(q, \dot{q})
$$

where $u$ is a new control input. Equating (3) and (15) yields

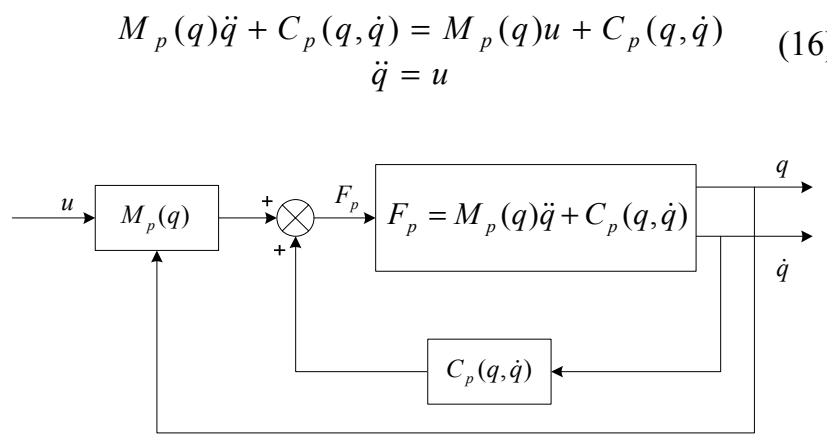

Fig. 3. Feedback linearization of the manipulator model.

Fig. 3 shows a block diagram of the linearized manipulator model. Let $\ddot{q}^{d}$ be the desired joint acceleration of the linear actuator and $v$ be the control output of the position feedback loop. In this case, the control input $u$ can be expressed as

$$
u=\ddot{q}^{d}+v
$$

A joint-based PID computed-torque controller is implemented to control the position of the planar manipulator. The PID controller is expressed in the form of

$$
v=K_{p} e+K_{i} \int e d t+K_{d} \dot{e}
$$

where $e$ is the position error between the command position $q^{d}$ of the linear actuator and the joint position $q$ acquired from the linear encoder. Substituting (17) into (16), the overall control law becomes

$$
F_{p}=M(q)\left[\ddot{q}^{d}+K_{p} e+K_{i} \int e d t+K_{d} \dot{e}\right]+C_{p}(q, \dot{q})
$$

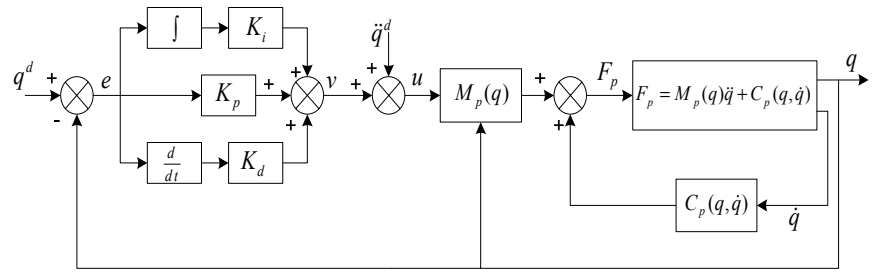

Fig. 4. Block diagram of the PID computed-torque controller.

Fig. 4 shows the block diagram of the proposed computed-torque PID controller. The closed-loop characteristic polynomial defined in [15] of the PID computed-torque controller described in (18) is

$$
\Delta_{c}(s)=\left|s^{3} I+K_{d} s^{2}+K_{p} s+K_{i}\right|
$$

and the diagonal control gains are defined as $K_{d}=$ $\operatorname{diag}\left\{K_{d m}\right\}, K_{p}=\operatorname{diag}\left\{K_{p m}\right\}, K_{i}=\operatorname{diag}\left\{K_{i m}\right\}$ ( $m=1$ and 2$)$. The damping ratio $\zeta$ and the natural frequency $\omega_{n}$ of the joint error $m$ are used for designing the control gains of the manipulator as

$$
K_{p m}=\omega_{n}^{2}, \quad K_{d m}=2 \zeta \omega_{n}, \quad K_{i m}<K_{d m} K_{p m}
$$

\section{PRototype Setup}

A prototype of the manipulator is designed and constructed as shown in Fig. 5. A typical BGA substrate is mounted on the end-effector to simulate the actual operation of the alignment process. The dimensions of the linkages are determined by kinematics optimization as described in [13]. The mechanical properties of the moving parts are then determined. Table I lists the dimensions and mechanical characteristics of the linkages of the manipulator, based on which a prototype has been constructed.

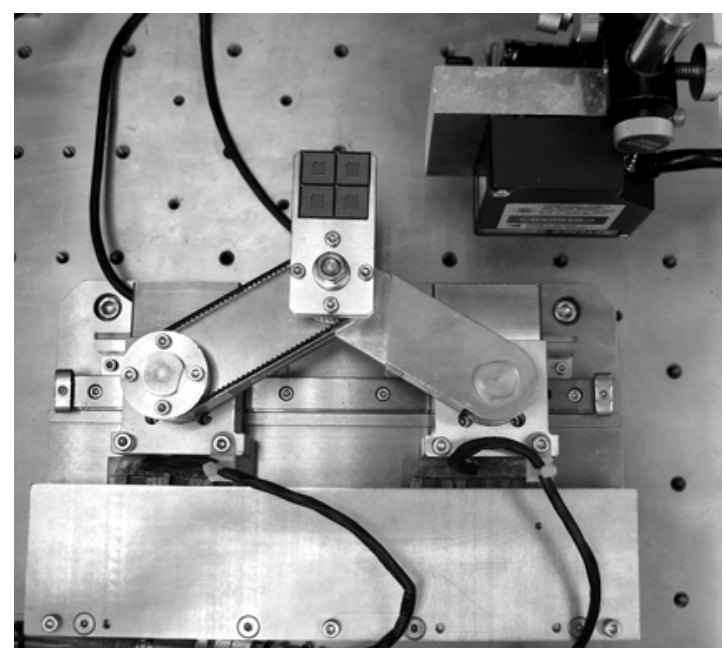

Fig. 5. Experimental setup of the planar manipulator prototype. 
TABLE I

KineMATICS AND DyNAMIC PROPERTIES OF THE MANIPULATOR

\begin{tabular}{ll}
\hline \hline Parameter & Value \\
\hline Actuator ranges $(\mathrm{mm})$ & \\
$d_{l \min }$ & 59.5 \\
$d_{\text {max }}$ & 99.5 \\
$d_{3 \min }$ & 119.5 \\
$d_{3 \max }$ & 159.5 \\
Length $(\mathrm{mm})$ & \\
$l_{2}$ and $l_{4}$ & 70 \\
$r_{2}$ & 43 \\
$r_{4}$ & 46 \\
$d_{y}$ & 10 \\
Mass $(\mathrm{kg})$ & \\
$m_{1}$ and $m_{3}$ & 0.7 \\
$m_{2}$ & 0.104 \\
$m_{4}$ & 0.094 \\
$m_{p}$ & 0.034 \\
Moment of inertia $\left(\mathrm{kgm}^{2}\right)$ & \\
$I_{2}$ & $7.2 \times 10^{-5}$ \\
$I_{4}$ & $5.5 \times 10^{-5}$ \\
\hline \hline
\end{tabular}

Four ironless linear brushless servo motors are used as actuators to drive the manipulator, and high precision linear encoders with $0.2 \mu \mathrm{m} /$ count resolution are used for measuring the actual positions of the prismatic joints of the parallel manipulator. In Fig. 5, a high precision laser displacement measurement system Keyence LB-041 with $0.91 \mu \mathrm{m} / \mathrm{mV}$ resolution is mounted on the fixed base of the manipulator to provide an independent measurement of the position of the end-effector. A PC-DSP control system is used to implement the motion controller of the manipulator.

\section{EXPERIMENTAL RESULTS}

A motion trajectory is designed to carry out a typical point-to-point motion of the manipulator for performing the substrate alignment process. The original location of the end-effector measured from the origin is $\left(x_{0}, y_{0}\right)=$ $(109.5 \mathrm{~mm}, 73.246 \mathrm{~mm})$ and the destination is $\left(x_{1}, y_{l}\right)=$ $(117 \mathrm{~mm}, 74.372 \mathrm{~mm})$. The required trajectory time of this motion is $70 \mathrm{~ms}$. The end-effector trajectory is mapped into two $5^{\text {th }}$ order polynomial motion profiles for the linear actuators using the inverse kinematics model and precomputed in the PC. The PID computed-torque controller designed in the section III is downloaded into the DSP platform for real-time control with sampling frequency of 2 $\mathrm{KHz}$. The required end-point accuracy of the end-effector to the destination point is $\pm 10 \mu \mathrm{m}$ in the XY plane.

Figs. 6 and 7 show the position and velocity tracking of the actuator $d_{1}$ compared with the $5^{\text {th }}$ order polynomial motion profiles generated in the joint space. The tracking performance of the actuator $d_{3}$ is shown in Figs. 8 and 9. The position errors of the actuators $d_{1}$ and $d_{3}$ as measured by the linear encoders in the joint space are shown in Fig. 10.

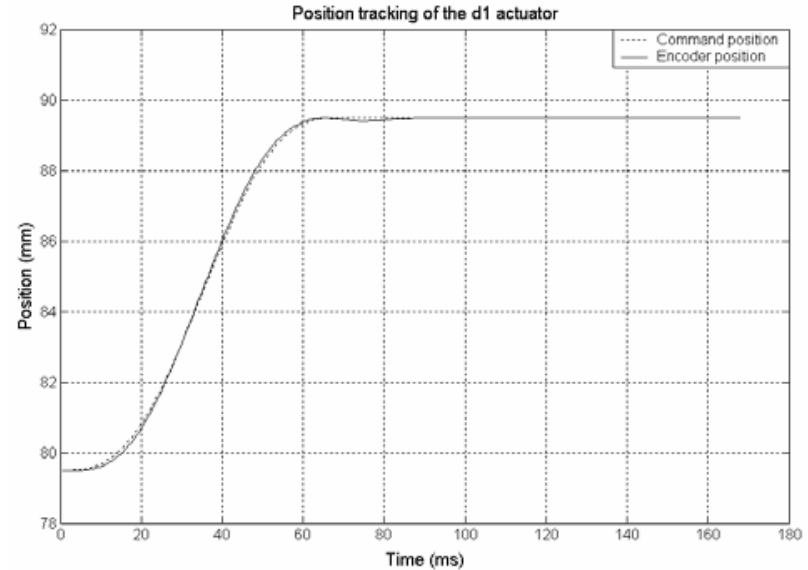

Fig. 6. Position tracking of the $d_{l}$ actuator.

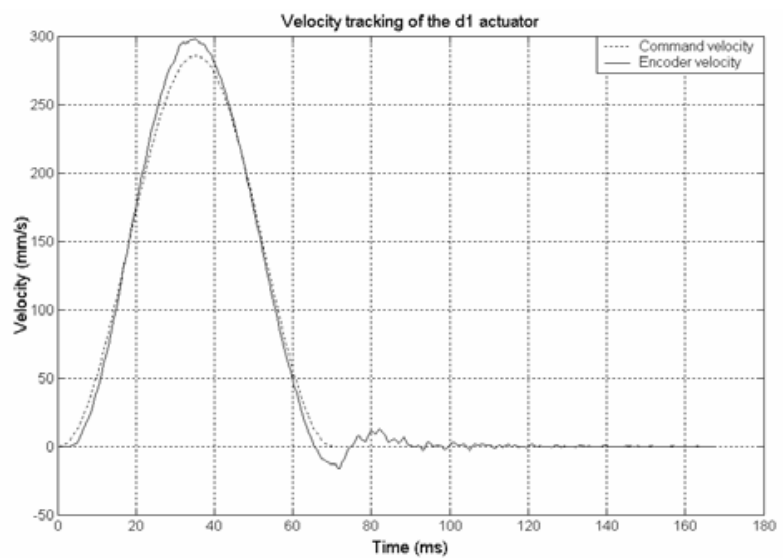

Fig. 7. Velocity tracking of the $d_{l}$ actuator.

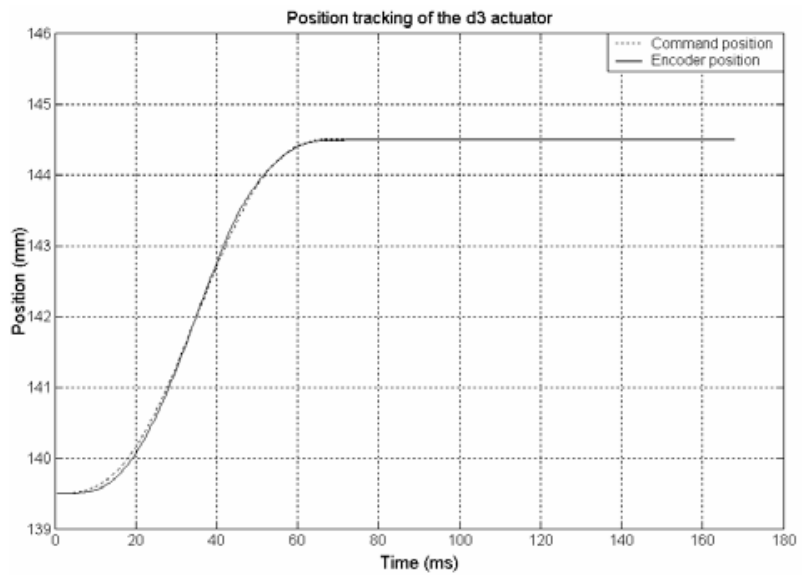

Fig. 8. Position tracking of the $d_{3}$ actuator. 


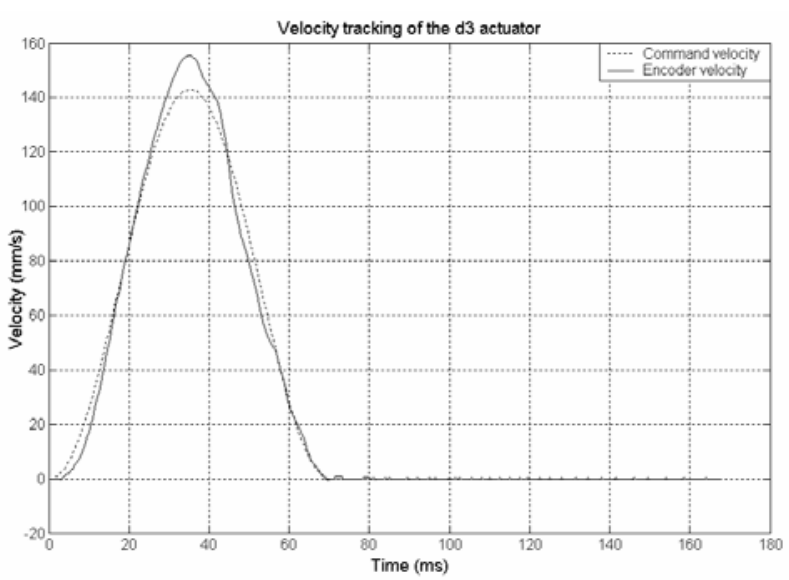

Fig. 9. Velocity tracking of the $d_{3}$ actuator.

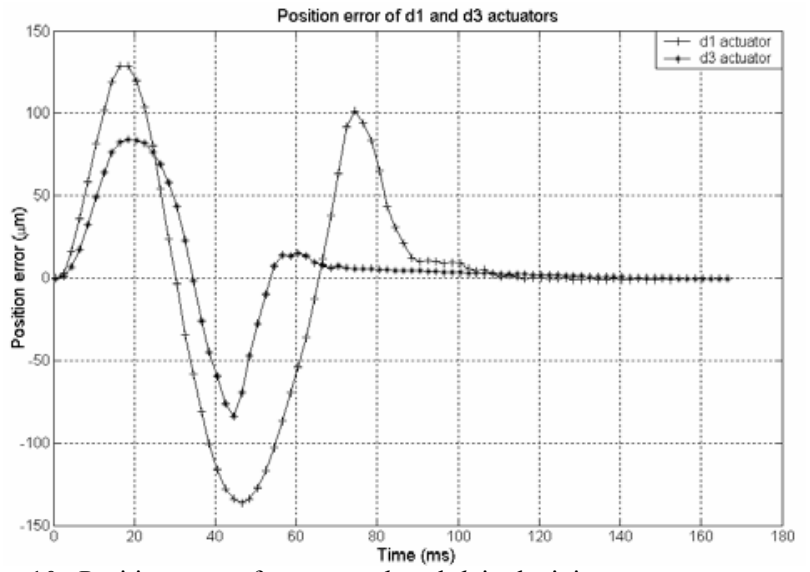

Fig. 10. Position error of actuators $d_{1}$ and $d_{3}$ in the joint space.

The desired and estimated travelling positions of the endeffector are determined using the forward kinematics model and plotted in Fig. 11. The estimated position errors of the end-effector in the $\mathrm{X}$ and $\mathrm{Y}$ directions are shown in Fig. 12.

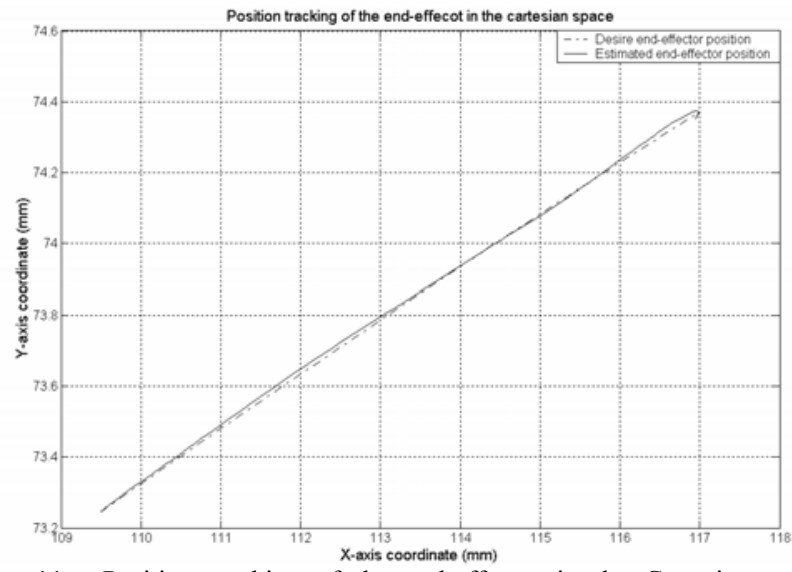

Fig. 11. Position tracking of the end-effector in the Cartesian space determined by the forward kinematics model.

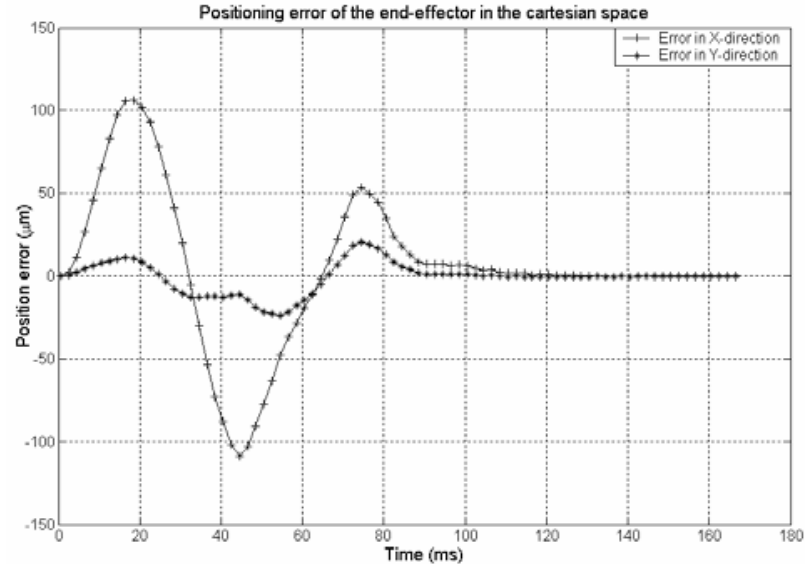

Fig. 12. Position error of the end-effector in the Cartesian space determined by the forward kinematics model.

Whether the end-effector achieves the desired end-point accuracy within the desired settling time are verified independently using a laser displacement measurement system. In Figs. 13 and 14, the X and Y positions of the endeffector is measured over an interval covering the end of motion profile of the linear actuator (dotted vertical line corresponds to $t=70 \mathrm{~ms}$ ) and the time when the position accuracy falls within $\pm 10 \mu \mathrm{m}$ (solid vertical line in Figs. 13 \& 14). The measurement results of the planar manipulator are compared with traditional $\mathrm{XY}$ motion stages and summarized in Table II. From Figs. 13 and 14, a significant reduction in the settling time of the end-effector is obtained as compared with the traditional XY motion stage given in Table II. The end-point accuracy demonstrates that the steady-state error of the parallel manipulator is significantly less than that of an XY stage.

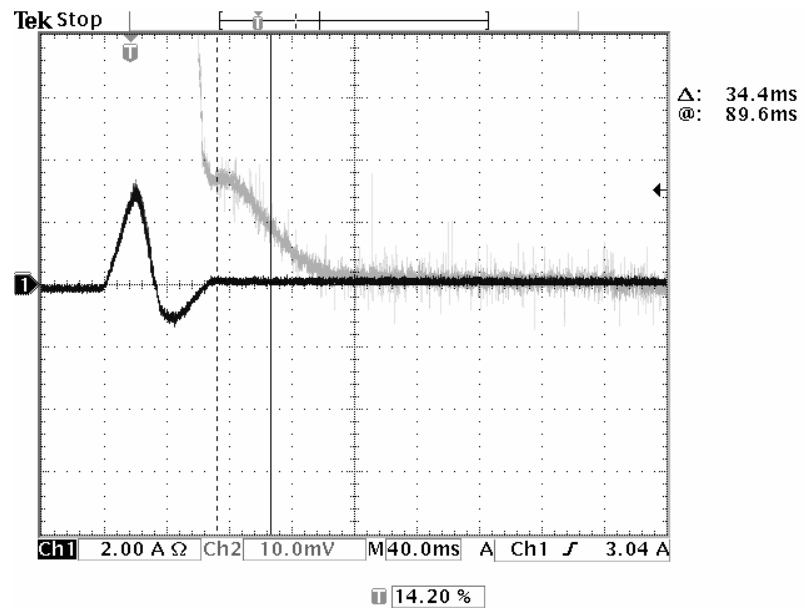

Fig. 13. Position response of the end-effector in X-direction over the settling interval measured by laser displacement system. 


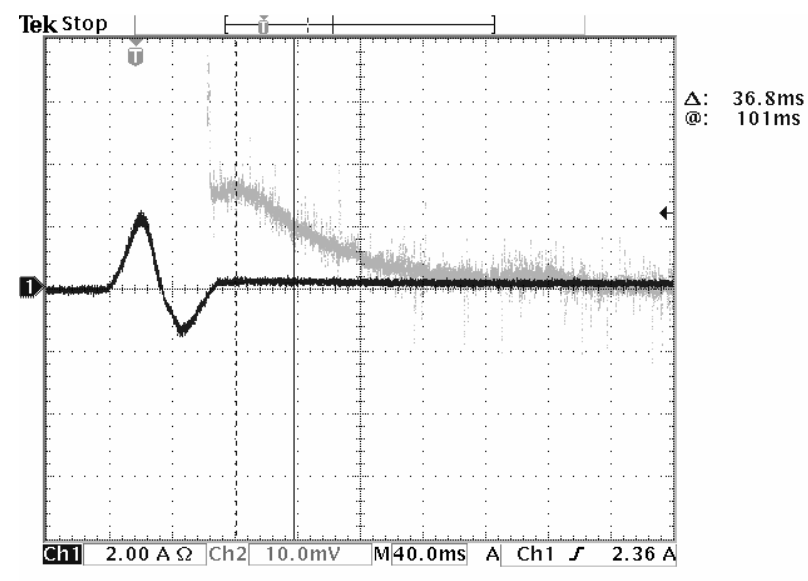

it $14.20 \%$

Fig. 14. Position response of the end-effector in Y-direction over the settling interval measured by laser displacement system.

TABLE II

ANALYSIS OF THE SETTLING TiME AND END-POINT ACCURACY OF THE PlanAR MANIPULATOR AND THE XY MOTION STAGE

\begin{tabular}{lll}
\hline \hline & $\begin{array}{c}\text { XY Motion } \\
\text { Stage }\end{array}$ & $\begin{array}{c}\text { Planar } \\
\text { Manipulator }\end{array}$ \\
\hline Settling time in X-direction $(\mathrm{ms})$ & 40.5 & 34.4 \\
Settling time in Y-direction $(\mathrm{ms})$ & 45.6 & 36.8 \\
End-point accuracy in X-dir. $(\mu \mathrm{m})$ & 6.0 & 1.82 \\
End-point accuracy in Y-dir. $(\mu \mathrm{m})$ & 8.0 & 2.73 \\
\hline \hline
\end{tabular}

TABLE III

COMPARISON OF THE MOVIng MASS AND the ACTUATOR POWER OF THE Planar MANIPULATOR AND THE XY STAGE

\begin{tabular}{lll}
\hline \hline & $\begin{array}{c}\text { XY Motion } \\
\text { Stage }\end{array}$ & $\begin{array}{c}\text { Planar } \\
\text { Manipulator }\end{array}$ \\
\hline Moving mass in X-direction (kg) & 8.2 & 0.97 \\
Moving mass in Y-direction (kg) & 3.0 & 0.97 \\
Required motor peak current (A) & 12 & 3 \\
Required actuator power (W) & 100 & 20 \\
\hline \hline
\end{tabular}

Table III shows the advantages of the proposed manipulator over the traditional XY motion stage in terms of the total moving mass, the required peak current and the required motor power Other benefits include a reduction in the size of the mechanism and savings in the actuator driving energy. A direct comparison also shows that the motion performance of our parallel mechanism is better than the 2DOF manipulator proposed in [8].

\section{CONCLUSION}

A significant improvement of the end-effector settling time and steady-state accuracy over the XY motion stage demonstrates the capability of the proposed planar parallel manipulator for high accuracy semiconductor packaging applications. The PID computed-torque controller implemented for the manipulator provides an effective control method to achieve high end-point positioning accuracy. The performance as measured by the highprecision laser displacement system provides a convincing and independent verification on the accuracy of the endeffector. Furthermore, the size of the manipulator and the driving energy of the actuators are less demanding than the tradition XY motion stage. The purpose of this study is to show that the proposed parallel manipulator is suitable for use as a superior alternative to the traditional XY motion stage on semiconductor packaging systems.

\section{ACKNOWLEDGMENT}

The authors gratefully acknowledge the technical help and advice received from Dr. P.C.K. Liu, Dr. G.P. Widdowson and Mr. S.K. Wong of ASM. This work is partially supported by a grant from the CRCG of The University of Hong Kong.

\section{REFERENCES}

[1] V. E. Gough, "Contribution to discussion of papers on research in automotive stability, control and tyre performance," Proc. Auto Div., Inst. Mechanical Engineers, 1956.

[2] D. Stewart, "A platform with 6 degree of freedom," Proc. of the Inst. of Mech. Engineers, 180 (Part 1, 15), 1965, pp.371-386.

[3] R. Clavel, "DELTA4, a fast robot with parallel geometry," Proc. International Symposium on Industrial Robots, 1998, pp. 92-100.

[4] P. Chiacchio, F. Pierrot, L. Sciavicco and B. Siciliano, "Robust design of independent joint controllers with experimentation on a high-speed parallel robot," IEEE Trans. Industrial Electronics, vol. 40, no. 4, pp.393-403, August 1993.

[5] G. W. Lee and F. T. Cheng, "Robust control of manipulators using the computed torque plus $H_{\infty}$ compensation method," Proc. IEE Control Theory Appl., vol.143. no.1, pp.64-72, January 1996.

[6] F. Lin and R. D. Brandt, "An optimal control approach to robust control of robot manipulators," IEEE Trans. Robotics and Automaton, vol.14, no.1, pp.69-77, February 1998.

[7] L. Gonzalez and L. Aguilar, " $H_{\infty}$ robust control design for an arm manipulator," J. Intelligent and Robotic System, vol. 27, pp.21-30, 2000.

[8] F. H. Ghorbel, O. Chetelat, R. Gunawardana and R. Longchamp, "Modeling and set point control of closed-chain mechanisms: theory and experiment," IEEE Trans. Control Systems Technology, vol. 8, no. 5, pp. 801-815, Sept. 2000.

[9] F. Marquet, S. Krut, O. Company and F. Pierrot, "ARCHI: a new redundant parallel mechanism - modeling, control and first results," Proc. IEEE/RSJ Int. Conf. Intelligent Robots and Systems, 2001, pp. 183-188.

[10] H. B. Choi, O. Company, F. Pierrot, A. Konno, T. Shibukawa and M. Uchiyama, "Design and control of a novel 4-DOFs parallel robot H4," Proc. IEEE Int. Conf. Robotics and Automation, 2003, pp. 1185-1190.

[11] A. Vivas, P. Poignet and F. Pierrot, "Predictive functional control for a parallel robot," Proc. IEEE/RSJ Int. Conf. Intelligent Robots and Systems, pp. 2785-2790, Oct. 2003.

[12] J. W. F. Cheung, Y. S. Hung and G. P. Widdowson, "Design and Analysis of a novel 4-dof parallel manipulator for semiconductor applications," The 8th Mechatronics Forum International Conference, 2002, pp. 1358-1366.

[13] J. W. F. Cheung and Y. S. Hung, "Kinematics optimization for high positioning accuracy of a 4-DOF parallel manipulator for semiconductor applications," in Proc. IEEE/ASME International Conference on Advanced Intelligent Mechatronics, Kobe, Japan, 2003, pp. 1256-1261.

[14] J. W. F. Cheung and Y. S. Hung, "Dynamic Modelling of a 4-DOF Parallel Manipulator for Semiconductor Packaging Systems," submitted to IEEE Trans. Automation Science and Engineering.

[15] F. L. Lewis, D. M. Dawson and C. T. Abdallah, Robot Manipulator Control Theory and Practice, $2^{\text {nd }}$ ed., New York: Marcel Dekker, 2004. 